\title{
The Poisson equation on Riemannian manifolds with weighted Poincaré inequality at infinity
}

\author{
Giovanni Catino $^{1} \cdot$ Dario D. Monticelli ${ }^{1} \cdot$ Fabio Punzo $^{1}$
}

Received: 2 September 2019 / Accepted: 26 June 2020 / Published online: 15 July 2020

(c) The Author(s) 2020

\begin{abstract}
We prove an existence result for the Poisson equation on non-compact Riemannian manifolds satisfying weighted Poincaré inequalities outside compact sets. Our result applies to a large class of manifolds including, for instance, all non-parabolic manifolds with minimal positive Green's function vanishing at infinity. On the source function, we assume a sharp pointwise decay depending on the weight appearing in the Poincaré inequality and on the behavior of the Ricci curvature at infinity. We do not require any curvature or spectral assumptions on the manifold. In comparison with previous works, we can deal with a more general setting on the curvature bounds and without any spectral assumption.
\end{abstract}

Keywords Poisson equation · Riemannian manifolds · Green's functions · Weighted Poincaré inequality

Mathematics Subject Classification $53 \mathrm{C} 21 \cdot 35 \mathrm{R} 01$

\section{Introduction}

The existence of solutions to the Poisson equation

$$
-\Delta u=f
$$

on a complete Riemannian manifold $(M, g)$, for a given function $f$ on $M$, is a classical problem which has been the object of deep interest in the literature. Malgrange [11] obtained solvability of the Poisson equation for any smooth function $f$ with compact support, as a consequence of the existence of a Green's function for $-\Delta$ on every complete Riemannian manifold. Under integrability assumptions on $f$, existence of solutions has been established

Fabio Punzo

fabio.punzo@polimi.it

Giovanni Catino

giovanni.catino@polimi.it

Dario D. Monticelli

dario.monticelli@polimi.it

1 Dipartimento di Matematica, Politecnico di Milano, Piazza Leonardo da Vinci 32, 20133 Milan, Italy 
by Strichartz [17] and Ni-Shi-Tam [16, Theorem 3.2] (see also [15, Lemma 2.3]). Moreover, in the same paper, the authors proved an existence result for the Poisson problem on manifolds with nonnegative Ricci curvature under a sharp integral assumption involving suitable averages of $f$. This condition in particular is satisfied if

$$
|f(x)| \leq \frac{C}{(1+r(x))^{\alpha}}
$$

for some $C>0$ and $\alpha>2$, where $r(x):=\operatorname{dist}(x, p)$ is the distance function of any $x \in M$ from a fixed reference point $p \in M$. In fact, they proved a more general result where the decay rate of $f$ is just assumed to be of order $1+\varepsilon$. Note that this result is sharp on the flat space $\mathbb{R}^{n}$.

From now on let us consider solutions $u$ of the Poisson equation $-\Delta u=f$ which can be represented as

$$
u(x)=\int_{M} G(x, y) f(y) \mathrm{d} y,
$$

where $G(x, y)$ is a Green's function of $-\Delta$ on $M$ (see Sect. 2 for further details). Muntenau-Sesum [12] addressed the case of manifolds with positive spectrum, i.e., $\lambda_{1}(M)>0$, and Ricci curvature bounded from below, obtaining existence of solutions under the pointwise decay assumption

$$
|f(x)| \leq \frac{C}{(1+r(x))^{\alpha}}
$$

for some $C>0$ and $\alpha>1$. Note that this result is sharp on $\mathbb{H}^{n}$. Their proof relies on very precise integral estimates on the minimal positive Green's function, which are inspired by the work of Li-Wang [10]. Note that in [12,13] the authors also study the behavior of the solution at infinity.

In [4] the authors generalized the existence result in [12], obtaining existence of solutions on manifolds with positive essential spectrum, i.e., $\lambda_{1}^{\text {ess }}(M)>0$, for source functions $f$ satisfying

$$
\sum_{m=1}^{\infty} \frac{\theta_{R}(m+1)-\theta_{R}(m)}{\lambda_{1}\left(M \backslash B_{m-1}(p)\right)} \sup _{M \backslash B_{m-1}(p)}|f|<\infty,
$$

for any $R>0$, where $\theta_{R}(m)$ is a function related to a lower bound on the Ricci curvature, locally on geodesic balls with center $p$ and radius $2 R+m$. In particular, the authors showed in [4, Corollary 1.3] existence of solutions on Cartan-Hadamard manifolds with strictly negative Ricci curvature, whenever

$$
-C(1+r(x))^{\gamma_{1}} \leq \text { Ric } \leq-\frac{1}{C}(1+r(x))^{\gamma_{2}}, \quad|f(x)| \leq \frac{C}{(1+r(x))^{\alpha}},
$$

for some $C>0$ and $\gamma_{1}, \gamma_{2} \geq 0$ with $\alpha>1+\frac{\gamma_{1}}{2}-\gamma_{2}$.

Observe that the results in $[4,12]$ cannot be used whenever the Ricci curvature tends to zero at infinity fast enough (see [19]) since, in this case, one has $\lambda_{1}^{\text {ess }}(M)=0$ (and so $\left.\lambda_{1}(M)=0\right)$. In particular, the case of $\mathbb{R}^{n}$ is not covered. On the other hand, the result in [16] does not apply on manifolds with negative curvature. The purpose of our paper is to obtain a general result which includes, as special cases, both manifolds with strictly 
negative curvature and manifolds with Ricci curvature vanishing at infinity. Moreover, our result is sharp on spherically symmetric manifolds, and in particular on $\mathbb{R}^{n}$ and $\mathbb{U}^{n}$.

Note that the condition $\lambda_{1}(M)>0$ is equivalent to the validity of the Poincare inequality

$$
\lambda_{1}(M) \int_{M} u^{2} \mathrm{~d} V \leq \int_{M}|\nabla u|^{2} \mathrm{~d} V
$$

for any $u \in C_{c}^{\infty}(M)$. On the other hand, one has positive essential spectrum if and only if, for some compact subset $K \subset M$, one has $\lambda_{1}(M \backslash K)>0$ and

$$
\lambda_{1}(M \backslash K) \int_{M} u^{2} \mathrm{~d} V \leq \int_{M}|\nabla u|^{2} \mathrm{~d} V
$$

for any $u \in C_{c}^{\infty}(M \backslash K)$. Generalizing the previous inequalities, one says that $(M, g)$ satisfies a weighted Poincaré inequality with a nonnegative weight function $\rho$ if

$$
\int_{M} \rho v^{2} \mathrm{~d} V \leq \int_{M}|\nabla v|^{2} \mathrm{~d} V
$$

for every $v \in C_{c}^{\infty}(M)$. If for any $R \geq R_{0}>0$, there exists a nonnegative function $\rho_{R}$ defined on $M$ such that (1) holds for every $v \in C_{c}^{\infty}\left(M \backslash B_{R}(p)\right)$ and for $\rho \equiv \rho_{R}$, we say that $(M, g)$ satisfies a weighted Poincaré inequality at infinity. In addition, inspired by [10], we say that $(M, g)$ satisfies the property $\left(\mathcal{P}_{w}^{\infty}\right)$, if a weighted Poincaré inequality at infinity holds for the family of weights $\rho_{R}$ and the conformal $\rho_{R}$-metric defined by

$$
g_{\rho_{R}}:=\rho_{R} g
$$

is complete for every $R \geq R_{0}$. The validity of a weighted Poincaré inequality on some classes of manifolds has been investigated in the literature. It is well known that on $\mathbb{R}^{n}$ inequality (1) holds with $\rho(x)=\frac{(n-2)^{2}}{4} \frac{1}{r^{2}(x)}$. It is also called Hardy inequality. More in general, it holds on every Cartan-Hadamard manifold with $\rho(x)=\frac{C}{r^{2}(x)}$, for some $C>0$ (see $[1,3]$ for some refinement of this result).

In order to state our main results, we need to introduce a (increasing) function $\omega(s)$ related to the value of the Ricci curvature on the annulus $B_{\frac{5}{4} s}(p) \backslash B_{\frac{3}{4} s}(p)$ (see (4) for the precise definition). In this paper, we prove the following result.

Theorem 1.1 Let $(M, g)$ be a complete non-compact Riemannian manifold satisfying the property $\left(\mathcal{P}_{w}^{\infty}\right)$ w.r.t. the family of weights $\rho_{R}, R \geq R_{0}$, and let $f$ be a locally Hölder continuous function on $M$. If

$$
\sum_{m}^{\infty}(\omega(m+1)-\omega(m)+1) \sup _{M \backslash B_{m}(p)} \frac{|f|}{\rho_{m}}<\infty,
$$

then the Poisson equation

$$
-\Delta u=f \quad \text { in } M
$$

admits a classical solution $u$.

Assume that $\lambda_{1}^{\text {ess }}(M)>0$ and 


$$
\text { Ric } \geq-C(1+r(x))^{\gamma}
$$

for some $\gamma \geq 0$. Then, it is direct to see that

$$
\omega(m+1)-\omega(m) \sim C\left(\theta_{R}(m+1)-\theta_{R}(m)\right) \sim C m^{\frac{\gamma}{2}}
$$

for every $R>0$ and the property $\left(\mathcal{P}_{w}^{\infty}\right)$ w.r.t. the family of weights $\rho_{R}, R \geq R_{0}$, holds for every $R$ with $\rho_{R}(x)=\lambda_{1}\left(M \backslash B_{R}(p)\right)$. Thus,

$$
(\omega(m+1)-\omega(m)+1) \sup _{M \backslash B_{m}(p)} \frac{|f|}{\rho_{m}} \sim C \frac{\theta_{R}(m+1)-\theta_{R}(m)}{\lambda_{1}\left(M \backslash B_{m}(p)\right)} \sup _{M \backslash B_{m}(p)}|f| ;
$$

therefore, our existence result is in accordance with those in $[4,12]$.

We recall that by [10, Corollary 1.4, Lemma 1.5] the validity of a weighted Poincare inequality (1) on $M$ implies the non-parabolicity of the manifold; on the contrary, if $(M, g)$ is non-parabolic, then a weighted Poincaré inequality holds on $M$, with weight

$$
\rho(x):=\frac{|\nabla G(p, x)|^{2}}{4 G^{2}(p, x)}
$$

where $G$ is the minimal positive Green's function on $(M, g)$. Exploiting this result, using similar techniques as in Theorem 1.1, we obtain the following refined result on complete non-compact non-parabolic manifolds.

Theorem 1.2 Let $(M, g)$ be a complete non-compact non-parabolic Riemannian manifold with minimal positive Green's function G. Let $\rho(x)=\frac{|\nabla G(p, x)|^{2}}{4 G^{2}(p, x)}$ and let $f$ be a locally Hölder continuous function on $M$. If

$$
\sum_{m}^{\infty}(\omega(m+1)-\omega(m)) \sup _{M \backslash B_{m}(p)} \frac{|f|}{\rho}<\infty,
$$

then the Poisson equation

$$
-\Delta u=f \quad \text { in } M
$$

admits a classical solution $u$.

Remark 1.3 We explicitly observe that in Theorem 1.2 the completeness of the conformal metric $g_{\rho}=\rho g$ is not required. As it was observed in [10], the completeness of $g_{\rho}$ would hold if $G(p, x) \rightarrow 0$ as $r(x) \rightarrow \infty$, a condition that we do not need to assume here.

It is well known that $\mathbb{R}^{n}$ is a non-parabolic manifold if $n \geq 3$, with minimal positive Green's function $G(x, y)=\frac{c_{n}}{|x-y|^{n-2}}$ for some positive constant $c_{n}$. Moreover, the weighted Poincaré - Hardy's inequality holds on $\mathbb{R}^{n}$ with

$$
\rho(x)=\frac{|\nabla G(0, x)|^{2}}{4 G^{2}(0, x)}=\frac{(n-2)^{2}}{4} \frac{1}{|x|^{2}} .
$$

In this case, using the definition (4) of the function $\omega(s)$, it is easy to see that 


$$
\omega(m+1)-\omega(m) \sim C \log \left(1+\frac{1}{m}\right) \sim \frac{C}{m} .
$$

Hence, we can apply Theorem 1.2, with

$$
(\omega(m+1)-\omega(m)) \sup _{M \backslash B_{m}(p)} \frac{|f|}{\rho_{m}} \sim C m \sup _{M \backslash B_{m}(p)}|f|
$$

and the convergence of the series follows, whenever $|f(x)| \leq C /(1+r(x))^{\alpha}$ for some $\alpha>2$. This condition is optimal, as it can be easily verified by explicit computations.

In general, concerning Cartan-Hadamard manifolds, by using Theorem 1.1 we improve [4, Corollary 1.3] allowing the Ricci curvature to approach zero at infinity.

Corollary 1.4 Let $(M, g)$ be a Cartan-Hadamard manifold, and let $f$ be a locally Hölder continuous, bounded function on $M$. If

$$
-C(1+r(x))^{\gamma_{1}} \leq \text { Ric } \leq-\frac{1}{C}(1+r(x))^{\gamma_{2}}, \quad|f(x)| \leq \frac{C}{(1+r(x))^{\alpha}},
$$

for some $C \geq 1, \gamma_{1}, \gamma_{2} \in \mathbb{R}, \gamma_{1} \geq \gamma_{2}, \gamma_{1} \geq 0$ and $\alpha$ satisfying

$$
\alpha>\left\{\begin{array}{ll}
1+\frac{\gamma_{1}}{2}-\gamma_{2} & \text { if } \gamma_{2} \geq-2 \\
3+\frac{\gamma_{1}}{2} & \text { if } \gamma_{2}<-2
\end{array},\right.
$$

then the Poisson equation

$$
-\Delta u=f \quad \text { in } M
$$

admits a classical solution $u$.

Remark 1.5 In the special case $\gamma_{1}=\gamma_{2}=\gamma \geq 0$ the condition on $\alpha$ in the previous corollary becomes

$$
\alpha> \begin{cases}1-\frac{\gamma}{2} & \text { if } \gamma \geq-2 \\ 2 & \text { if } \gamma<-2\end{cases}
$$

In particular, in $(M, g)$ is the standard hyperbolic space $\mathbb{U}^{n}$, and then $\gamma=0$. Thus, we need that $\alpha>1$ and this condition is sharp as observed above. We will consider also the case $\gamma<0$ in Sect. 6.2 on model manifolds.

The paper is organized as follows: In Sect. 2 we collect some preliminary results and we define precisely the function $\omega$; in Sect. 3 we prove a refined local gradient estimates for positive harmonic functions; in Sect. 4 we prove key estimates on the positive minimal Green's function $G(x, y)$ of a non-parabolic manifold, by means of the property $\left(\mathcal{P}_{w}^{\infty}\right)$ w.r.t. the family of weights $\rho_{R}, R \geq R_{0}$; in Sect. 5 we prove Theorem 1.1 ; finally, in Sect. 6 we prove Corollary 1.4 and show the optimality of the assumption in Theorem 1.2 for rotationally symmetric manifolds.

Finally, we note that some results concerning the Poisson equation on some manifolds satisfying a weighted Poincaré inequality have been very recently obtained in [14]. However, their assumptions and results apparently are completely different to ours. 


\section{Preliminaries}

Let $(M, g)$ be a complete non-compact $n$-dimensional Riemannian manifold. For any $x \in M$ and $R>0$, we denote by $B_{R}(x)$ the geodesic ball of radius $\mathrm{R}$ with center $x$ and let $\operatorname{Vol}\left(B_{R}(x)\right)$ be its volume. We denote by Ric the Ricci curvature of $g$. For any $x \in M$, let $\mu(x)$ be the smallest eigenvalue of Ric at $x$. Thus, for any $V \in T_{x} M$ with $|V|=1, \operatorname{Ric}(V, V)(x) \geq \mu(x)$ and we have $\mu(x) \geq-\omega(r(x))$ for some $\omega \in C([0, \infty)), \omega \geq 0$. Hence, for any $x \in M$, we have

$$
\operatorname{Ric}(V, V)(x) \geq-(n-1) \frac{\varphi^{\prime \prime}(r(x))}{\varphi(r(x))}
$$

for some $\varphi \in C^{\infty}((0, \infty)) \cap C^{1}([0, \infty))$ with $\varphi(0)=0$ and $\varphi^{\prime}(0)=1$. Note that $\varphi, \varphi^{\prime}, \varphi^{\prime \prime}$ are positive in $(0, \infty)$. We set

$$
K_{R}(x):=\sup _{y \in B_{r(x)+R}(p) \backslash B_{r(x)-R}(p)} \frac{\varphi^{\prime \prime}(r(y))}{\varphi(r(y))}
$$

for $r(x)>R>1$;

$$
\begin{aligned}
I_{R}(x):= & \begin{cases}\sqrt{K_{R}(x)} \operatorname{coth}\left(\sqrt{K_{R}(x)} R / 2\right) & \text { if } K_{R}(x)>0 \\
\frac{2}{R} & \text { if } K_{R}(x)=0 ;\end{cases} \\
& Q_{R}(x):=\max \left\{K_{R}(x), \frac{I_{R}(x)}{R}, \frac{1}{R^{2}}\right\} .
\end{aligned}
$$

Note that $Q_{R}(x) \equiv Q_{R}(r(x))$. For any $z \in M$, let $\gamma$ be the minimal geodesic connecting $p$ to $z$. We define the function

$$
\omega(z)=\omega(r(z)):=\int_{a}^{r(z)} \sqrt{Q_{\frac{r(\gamma(s)}{4}}(r(\gamma(s))} \mathrm{d} s,
$$

for a given $a>0$. Note that $t \mapsto \omega(t)$ is increasing and so invertible.

Under (2), we know that

$$
\operatorname{Vol}\left(B_{R}(p)\right) \leq C \int_{0}^{R} \varphi^{n-1}(\xi) \mathrm{d} \xi .
$$

Moreover, let $\operatorname{Cut}(p)$ be the cut locus of $p \in M$.

It is known that every complete Riemannian manifold admits a Green's function (see [11]), i.e., a smooth function defined in $(M \times M) \backslash\{(x, y) \in M \times M: x=y\}$ such that $G(x, y)=G(y, x)$ and $\Delta_{y} G(x, y)=-\delta_{x}(y)$. We say that $(M, g)$ is non-parabolic if there exists a minimal positive Green's function $G(x, y)$ on $(M, g)$, and parabolic otherwise.

We say that $(M, g)$ satisfies a weighted Poincaré inequality with a nonnegative weight function $\rho$ if

$$
\int_{M} \rho v^{2} \mathrm{~d} V \leq \int_{M}|\nabla v|^{2} \mathrm{~d} V
$$


for every $v \in C_{c}^{\infty}(M)$. If for any $R \geq R_{0}>0$, there exists a nonnegative function $\rho_{R}$ such that (1) holds for every $v \in C_{c}^{\infty}\left(M \backslash B_{R}(p)\right)$ and for $\rho \equiv \rho_{R}$, we say that $(M, g)$ satisfies a weighted Poincaré inequality at infinity. In addition, inspired by [10], we say that $(M, g)$ satisfies the property $\left(\mathcal{P}_{\rho_{R}}^{\infty}\right)$ if a weighted Poincaré inequality at infinity holds for the family of weights $\rho_{R}$ and the conformal $\rho_{R}$-metric defined by

$$
g_{\rho}:=\rho_{R} g
$$

is complete. With this metric we consider the $\rho$-distance function

$$
r_{\rho}(x, y)=\inf _{\gamma} l_{\rho}(\gamma)
$$

where the infimum of the lengths is taken over all curves joining $x$ and $y$, with respect to the metric $g_{\rho}$. For the fixed reference point $p \in M$, we denote by

$$
r_{\rho}(x)=r_{\rho}(p, x) \text {. }
$$

Note that $\left|\nabla r_{\rho}(x)\right|^{2}=\rho(x)$. Finally, we denote by

$$
B_{R}^{\rho}(p)=\left\{x \in M: r_{\rho}(x) \leq R\right\} .
$$

Let $\lambda_{1}(M)$ be the bottom of the $L^{2}$-spectrum of $-\Delta$. It is known that $\lambda_{1}(M) \in[0,+\infty)$ and it is given by the variational formula

$$
\lambda_{1}(M)=\inf _{v \in C_{c}^{\infty}(M)} \frac{\int_{M}|\nabla v|^{2} \mathrm{~d} V}{\int_{M} v^{2} \mathrm{~d} V} .
$$

If $\lambda_{1}(M)>0$, then $(M, g)$ is non-parabolic (see [6, Proposition 10.1]). Whenever $(M, g)$ is non-parabolic, let $G_{R}(x, y)$ be the Green's function of $-\Delta$ in $B_{R}(z)$ satisfying zero Dirichlet boundary conditions on $\partial B_{R}(z)$, for some $z \in M$. We have that $R \mapsto G_{R}(x, y)$ is increasing and, for any $x, y \in M$,

$$
G(x, y)=\lim _{R \rightarrow \infty} G_{R}(x, y),
$$

locally uniformly in $(M \times M) \backslash\{(x, y) \in M \times M: x=y\}$. We define $\lambda_{1}(\Omega)$, with $\Omega$ an open subset of $M$, to be the first eigenvalue of $-\Delta$ in $\Omega$ with zero Dirichlet boundary conditions. It is well known that $\lambda_{1}(\Omega)$ is decreasing with respect to the inclusion of subsets. In particular, $R \mapsto \lambda_{1}\left(B_{R}(x)\right)$ is decreasing and $\lambda_{1}\left(B_{R}(x)\right) \rightarrow \lambda_{1}(M)$ as $R \rightarrow \infty$.

For any $x \in M$, for any $s>0$ and for any $0 \leq a<b \leq+\infty$, we define

$$
\begin{aligned}
\mathcal{L}_{x}(s) & :=\{y \in M: G(x, y)=s\}, \\
\mathcal{L}_{x}(a, b) & :=\{y \in M: a<G(x, y)<b\} .
\end{aligned}
$$

\section{Local gradient estimate for harmonic functions}

In this section, we improve [4, Lemma 3.1]. We set

$$
k_{R}(z):=\sup _{B_{R}(z)} \frac{\varphi^{\prime \prime}(r(y))}{\varphi(r(y))}
$$


for $z \in M$ and $R>0$;

$$
i_{R}(z):= \begin{cases}\sqrt{k_{R}} \operatorname{coth}\left(\sqrt{k_{R}(z)} R / 2\right) & \text { if } k_{R}(z)>0 \\ \frac{2}{R} & \text { if } k_{R}(z)=0 .\end{cases}
$$

Lemma 3.1 Let $R>0$ and $z \in M$. Let $u \in C^{2}\left(B_{R}(z)\right)$ be a positive harmonic function in $B_{R}(z)$. Then,

$$
|\nabla u(\xi)| \leq C \sqrt{\max \left\{k_{R}(z), \frac{i_{R}(z)}{R}, \frac{1}{R^{2}}\right\}} u(\xi) \text { for any } \xi \in B_{R / 2}(z),
$$

for some positive constant $C>0$.

Proof Following the classical argument of Yau, let $v:=\log u$. Then,

$$
\Delta v=-|\nabla v|^{2} .
$$

Let $\eta(\xi)=\eta(d(\xi))$, with $d(\xi):=\operatorname{dist}(\xi, z)$, a smooth cutoff function such that $\eta(\xi) \equiv 1$ on $B_{R / 2}(z)$, with support in $B_{R}(z), 0 \leq \eta \leq 1$ and

$$
-\frac{4}{R} \leq \frac{\eta^{\prime}}{\eta^{1 / 2}} \leq 0 \quad \text { and } \quad \frac{\left|\eta^{\prime \prime}\right|}{\eta} \leq \frac{8}{R^{2}}
$$

Let $w=\eta^{2}|\nabla v|^{2}$. Then,

$$
\frac{1}{2} \Delta w=\frac{1}{2} \eta^{2} \Delta|\nabla v|^{2}+\frac{1}{2}|\nabla v|^{2} \Delta \eta^{2}+\left\langle\nabla|\nabla v|^{2}, \nabla \eta^{2}\right\rangle .
$$

Then, from classical Bochner-Weitzenböch formula and Newton inequality, one has

$$
\begin{aligned}
\frac{1}{2} \Delta|\nabla v|^{2} & =\left|\nabla^{2} v\right|^{2}+\operatorname{Ric}(\nabla v, \nabla v)+\langle\nabla v, \nabla \Delta v\rangle \\
& \geq \frac{1}{n}(\Delta v)^{2}-(n-1) \frac{\varphi^{\prime \prime}}{\varphi}|\nabla v|^{2}-\left\langle\nabla|\nabla v|^{2}, \nabla v\right\rangle \\
& =\frac{1}{n}|\nabla v|^{4}-(n-1) \frac{\varphi^{\prime \prime}}{\varphi}|\nabla v|^{2}-\left\langle\nabla|\nabla v|^{2}, \nabla v\right\rangle .
\end{aligned}
$$

Moreover, by Laplacian comparison, since Ric $\geq-(n-1) k_{R}(z)$ in $B_{R}(z)$, we have

$$
\begin{aligned}
\frac{1}{2} \Delta \eta^{2} & =\eta \eta^{\prime} \Delta \rho+\eta \eta^{\prime \prime}+\left(\eta^{\prime}\right)^{2} \\
& \geq(n-1) i_{R}(z) \eta \eta^{\prime}+\eta \eta^{\prime \prime}+\left(\eta^{\prime}\right)^{2} \\
& \geq-\frac{4}{R}\left((n-1) i_{R}(z)+\frac{2}{R}\right) \eta
\end{aligned}
$$

pointwise in $B_{R}(z) \backslash(\{z\} \cup \operatorname{Cut}(z))$ and weakly on $B_{R}(z)$. Thus, 


$$
\begin{aligned}
\frac{1}{2} \Delta w \geq & \frac{1}{n} \frac{w^{2}}{\eta^{2}}-(n-1) \frac{\varphi^{\prime \prime}}{\varphi} w-\frac{4}{R}\left((n-1) i_{R}(z)+\frac{2}{R}\right) \frac{w}{\eta} \\
& -4 \frac{\left|\eta^{\prime}\right|^{2}}{\eta^{2}} w+\frac{2}{\eta}\langle\nabla w, \nabla \eta\rangle-\langle\nabla w, \nabla v\rangle+\frac{2}{\eta}\langle\nabla v, \nabla \eta\rangle w \\
\geq & \frac{1}{n} \frac{w^{2}}{\eta^{2}}-(n-1) \frac{\varphi^{\prime \prime}}{\varphi} w-\frac{4}{R}\left((n-1) i_{R}(z)+\frac{2}{R}\right) \frac{w}{\eta} \\
& +\frac{2}{\eta}\langle\nabla w, \nabla \eta\rangle-\langle\nabla w, \nabla v\rangle-\frac{64}{R^{2}} \frac{w}{\eta}-\frac{8}{R} \frac{w^{3 / 2}}{\eta^{3 / 2}} \\
\geq & \frac{1}{2 n} \frac{w^{2}}{\eta^{2}}-(n-1) \frac{\varphi^{\prime \prime}}{\varphi} w-\frac{4}{R}\left((n-1) i_{R}(z)+\frac{18+8 n}{R}\right) \frac{w}{\eta} \\
& +\frac{2}{\eta}\langle\nabla w, \nabla \eta\rangle-\langle\nabla w, \nabla v\rangle .
\end{aligned}
$$

Let $q$ be a maximum point of $w$ in $\bar{B}_{R}(z)$. Since $w \equiv 0$ on $\partial B_{R}(z)$, we have $q \in B_{R}(z)$. First assume $q \notin \operatorname{Cut}(z)$. At $q$, we obtain

$$
0 \geq\left[\frac{1}{2 n} w-(n-1) \frac{\varphi^{\prime \prime}}{\varphi}-\frac{4}{R}\left((n-1) i_{R}(z)+\frac{18+8 n}{R}\right)\right] w .
$$

So

$$
w(q) \leq 2 n(n-1) \frac{\varphi^{\prime \prime}(r(q))}{\varphi(r(q))}+\frac{8 n(n-1)}{R} i_{R}(z)+\frac{144 n+64 n^{2}}{R^{2}} .
$$

Thus, for any $\xi \in B_{R / 2}(z)$,

$$
\begin{aligned}
|\nabla v(\xi)|^{2} & \leq 2 n(n-1) \frac{\varphi^{\prime \prime}(r(q))}{\varphi(r(q))}+\frac{8 n(n-1)}{R} i_{R}(z)+\frac{144 n+64 n^{2}}{R^{2}} \\
& \leq 2 n(n-1) k_{R}(z)+\frac{8 n(n-1)}{R} i_{R}(z)+\frac{144 n+64 n^{2}}{R^{2}}
\end{aligned}
$$

We get

$$
\frac{|\nabla u(\xi)|}{u(\xi)}=|\nabla v(\xi)| \leq C \sqrt{\max \left\{k_{R}(z), \frac{i_{R}(z)}{R}, \frac{1}{R^{2}}\right\}} .
$$

for some positive constant $C>0$. By standard Calabi trick (see $[2,5]$ ), the same estimate can be obtained when $q \in \operatorname{Cut}(z)$. This concludes the proof of the lemma.

As a corollary, we have the following

Corollary 3.2 Let $(M, g)$ be non-parabolic. If $r(z)>R>0$, then

$$
|\nabla G(p, z)| \leq C \sqrt{Q_{R}(z)} G(p, z),
$$

for some positive constant $C>0$. 


\section{Green's function estimates}

\subsection{Pointwise estimate}

Lemma 4.1 Let $(M, g)$ be non-parabolic, and let $a>0$ and $y \in M \backslash B_{a}(p)$. Then,

$$
A^{-1} \exp (-B \omega(y)) \leq G(p, y) \leq A \exp (B \omega(y)),
$$

where $A:=\max \left\{\max _{\partial B_{a}(p)} G(p, \cdot),\left(\min _{\partial B_{a}(p)} G(p, \cdot)\right)^{-1}\right\}$ and $B>0$ is a positive constant depending on $C$ from Corollary 3.2.

Proof Let $y \in M \backslash \overline{B_{a}(p)}$ with $a>0$ and consider the minimal geodesic $\gamma$ joining $p$ to $y$ and let $y_{0} \in \partial B_{a}(p)$ be a point of intersection of $\gamma$ with $\partial B_{a}(p)$. Since $G(p, \cdot)$ is harmonic in $B_{r(z) / 4}(z)$, for every $z \in \gamma$ with $r(z) \geq a$, by Corollary 3.2 we get

$$
|\nabla G(p, z)| \leq C \sqrt{Q_{r(z) / 4}(z)} G(p, z) .
$$

We have

$$
\begin{aligned}
G(p, y) & =G\left(p, y_{0}\right)+\int_{a}^{r(y)}\langle\nabla G(p, \gamma(s)), \dot{\gamma}(s)\rangle \mathrm{d} s \\
& \leq G\left(p, y_{0}\right)+C \int_{a}^{r(y)} \sqrt{Q_{\frac{r(\gamma(s))}{4}}(r(\gamma(s)))} G(p, \gamma(s)) \mathrm{d} s .
\end{aligned}
$$

By Gronwall's inequality,

$$
G(p, y) \leq G\left(p, y_{0}\right) \exp \left(C \int_{a}^{r(y)} \sqrt{Q_{\frac{r(\gamma(s))}{4}}(r(\gamma(s)))} \mathrm{d} s\right) \leq A \exp (B \omega(y)),
$$

with $A:=\max \left\{\max _{\partial B_{a}(p)} G(p, \cdot),\left(\min _{\partial B_{a}(p)} G(p, \cdot)\right)^{-1}\right\}$ and $B=C$. Similarly,

$$
G(p, y) \geq A^{-1} \exp (-B \omega(y)) \text {. }
$$

Remark 4.2 One has

$$
G(p, y) \geq A^{-1} \exp (-B \omega(a))
$$

for any $y \in \overline{B_{a}(p)}$. This follows from Lemma 4.1 with $y \in \partial B_{a}(p)$ and the maximum principle, since $y \mapsto G(p, y)$ is (weakly) superharmonic in $B_{a}(p)$. In particular,

$$
\mathcal{L}_{p}\left(0, A^{-1} \exp (-B \omega(a))\right) \subset M \backslash B_{a}(p) .
$$

Remark 4.3 We also note that

$$
\mathcal{L}_{p}(A \exp (B \omega(a)), \infty) \subset B_{a}(p) .
$$

In fact, let $y \in M \backslash B_{a}(p)$ and take $j>r(y)$. Since $G_{j}(p, y) \leq G(p, y)$ and $G_{j}(p, \cdot) \equiv 0$ on $\partial B_{j}(p)$, by Lemma 4.1, we have 


$$
G_{j}(p, y) \leq A \exp (B \omega(a)) \quad \text { on } \quad \partial\left(B_{j}(p) \backslash B_{a}(p)\right) ;
$$

note that the right-hand side is independent of $y$. Since $y \mapsto G_{j}(x, y)$ is harmonic in $B_{j}(p) \backslash B_{a}(p)$, by maximum principle,

$$
G_{j}(p, y) \leq A \exp (B \omega(a)) \quad \text { in } \quad B_{j}(p) \backslash B_{a}(p) .
$$

Sending $j \rightarrow \infty$, by (7), we obtain

$$
G(p, y) \leq A \exp (B \omega(a)) \quad \text { in } \quad M \backslash B_{a}(p),
$$

and the claim follows.

\subsection{Auxiliary estimates}

Lemma 4.4 Let $(M, g)$ be non-parabolic. For any $s>0$, there holds

$$
\int_{\mathcal{L}_{p}(s)}|\nabla G(p, y)| \mathrm{d} A(y)=1
$$

where $\mathrm{d} A(y)$ is the $(n-1)$-dimensional Hausdorff measure on $\mathcal{L}_{x}(s)$. As a consequence, by the co-area formula, for any $0<a<b$, there holds

$$
\int_{\mathcal{L}_{p}(a, b)} \frac{|\nabla G(p, y)|^{2}}{G(p, y)} \mathrm{d} y=\log \left(\frac{b}{a}\right) .
$$

For the proof see [12]. Moreover, we get the following weighted integrability property for the Green's function.

Lemma 4.5 Assume that $(M, g)$ satisfies the property $\left(\mathcal{P}_{w}^{\infty}\right)$ w.r.t. the family of weights $\rho_{R}$, $R \geq R_{0}$. Fix $m \geq R_{0}$. Then, for any $R_{1}>0$ such that $B_{m}(p) \subset B_{R_{1}}^{\rho_{m}}(p)$, one has

$$
\int_{M \backslash B_{2 R_{1}}^{\rho_{m}}(p)} \rho_{m}(y)|G(p, y)|^{2} \mathrm{~d} y<\infty .
$$

Remark 4.6 Note that $B_{m}(p) \subset B_{R_{1}}^{\rho_{m}}(p)$ for every $R_{1}$ large enough.

Proof In order to simplify the notation, let $\rho \equiv \rho_{m}$. Fix $R_{1}>0$ such that $B_{m}(p) \subset B_{R_{1}}^{\rho}(p)$ and let $\phi$ be defined as

$$
\phi(x):= \begin{cases}0 & \text { on } B_{R_{1}}^{\rho}(p) \\ \frac{r_{\rho}(x)-R_{1}}{R_{1}} & \text { on } B_{2 R_{1}}^{\rho}(p) \backslash B_{R_{1}}^{\rho}(p) \\ 1 & \text { on } M \backslash B_{2 R_{1}}^{\rho}(p) .\end{cases}
$$

Let $R>2 R_{1}$ and $G_{R}^{\rho}(p, y)$ be the Green's function of $-\Delta$ in $B_{R}^{\rho}(p)$ satisfying zero Dirichlet boundary conditions on $\partial B_{R}^{\rho}(p)$. Following the proof in [10], since $G_{R}^{\rho}$ is harmonic in $B_{R}^{\rho}(p)$, one has 


$$
\begin{aligned}
\int_{B_{R}^{\rho}(p)}\left|\nabla\left(\phi G_{R}^{\rho}\right)\right|^{2} \mathrm{~d} V= & \int_{B_{R}^{\rho}(p)}|\nabla \phi|^{2}\left(G_{R}^{\rho}\right)^{2} \mathrm{~d} V+\int_{B_{R}^{\rho}(p)}\left|\nabla G_{R}^{\rho}\right|^{2} \phi^{2} \mathrm{~d} V \\
& +2 \int_{B_{R}^{\rho}(p)}\left\langle\nabla \phi, \nabla G_{R}^{\rho}\right\rangle \phi G_{R}^{\rho} \mathrm{d} V \\
= & \int_{B_{R}^{\rho}(p)}|\nabla \phi|^{2}\left(G_{R}^{\rho}\right)^{2} \mathrm{~d} V+\frac{1}{2} \int_{B_{R}^{\rho}(p)} \Delta\left(G_{R}^{\rho}\right)^{2} \phi^{2} \mathrm{~d} V \\
& +2 \int_{B_{R}^{\rho}(p)}\left\langle\nabla \phi, \nabla G_{R}^{\rho}\right\rangle \phi G_{R}^{\rho} \mathrm{d} V \\
= & \int_{B_{R}^{\rho}(p)}|\nabla \phi|^{2}\left(G_{R}^{\rho}\right)^{2} \mathrm{~d} V
\end{aligned}
$$

where the last equality follows by integration by parts and the fact that $G_{R}^{\rho}(p, y)$ vanishes on $\partial B_{R}^{\rho}(p)$. Hence, the weighted Poincaré inequality yields

$$
\int_{M \backslash B_{R_{1}}^{\rho}(p)} \rho\left(G_{R}^{\rho}\right)^{2} \phi^{2} \mathrm{~d} V \leq \int_{B_{R}^{\rho}(p)}\left|\nabla\left(\phi G_{R}^{\rho}\right)\right|^{2} \mathrm{~d} V \leq \frac{1}{R_{1}^{2}} \int_{B_{2 R_{1}}^{\rho}(p) \backslash B_{R_{1}}^{\rho}(p)} \rho\left(G_{R}^{\rho}\right)^{2} \mathrm{~d} V
$$

Letting $R \rightarrow \infty$, by Fatou's lemma and uniform convergence of $G_{R}^{\rho} \rightarrow G$ on compact subsets, we get

$$
\int_{M \backslash B_{2 R_{1}}^{\rho}(p)} \rho G^{2} \mathrm{~d} V \leq \frac{1}{R_{1}^{2}} \int_{B_{2 R_{1}}^{\rho}(p) \backslash B_{R_{1}}^{\rho}(p)} \rho G^{2} \mathrm{~d} V
$$

and the thesis follows.

We expect a decay estimate similar to the one in [10, Theorem 2.1]. However, we leave out this refinement since it is not necessary in our arguments.

\subsection{Integral estimates on level sets}

We begin by noting that using Remark 4.3 and the fact that $G(p, \cdot) \in L_{\text {loc }}^{1}(M)$ one has the following integral estimate on large level sets.

Proposition 4.7 Let $(M, g)$ be non-parabolic. Choose A, B as in Lemma 4.1. Then,

$$
\int_{\mathcal{L}_{p}(A \exp (B \omega(a)), \infty)} G(p, y) \mathrm{d} y<\infty .
$$

For intermediate levels sets, we get the following key inequality.

Proposition 4.8 Assume that $(M, g)$ satisfies the property $\left(\mathcal{P}_{w}^{\infty}\right)$ w.r.t. the family of weights $\rho_{R}, R \geq R_{0}$. Then, there exists a positive constant $C$ such that for any function $f$ and any $0<\delta<1, \varepsilon>0$ satisfying $\mathcal{L}_{p}\left(\frac{\delta \varepsilon}{2}, 2 \varepsilon\right) \subset M \backslash B_{m}(p)$ for some $m>R_{0}$, one has

$$
\left|\int_{\mathcal{L}_{p}(\delta \varepsilon, \varepsilon)} G(p, y) f(y) \mathrm{d} y\right| \leq C(-\log \delta+1) \sup _{\mathcal{L}_{p}(\delta \varepsilon, \varepsilon)} \frac{|f|}{\rho_{m}} .
$$


Proof We follow the general argument in $[10,12]$; however, some relevant differences are in order, due to the use of the property $\left(\mathcal{P}_{w}^{\infty}\right)$. Let $\phi:=\chi \psi$ with

$$
\chi(y):= \begin{cases}\frac{1}{\log 2} \log \left(\frac{2 G(p, y)}{\delta \epsilon}\right) & \text { on } \mathcal{L}_{p}\left(\frac{\delta \varepsilon}{2}, \delta \varepsilon\right) \\ 1 & \text { on } \mathcal{L}_{p}(\delta \varepsilon, \varepsilon) \\ \frac{1}{\log 2} \log \left(\frac{2 \varepsilon}{G(p, y)}\right) & \text { on } \mathcal{L}_{p}(\varepsilon, 2 \varepsilon) \\ 0 & \text { elsewhere }\end{cases}
$$

and for any fixed $R>0$

$$
\psi(y):= \begin{cases}1 & \text { on } B_{R}^{\rho_{m}}(p) \\ R+1-r_{\rho_{m}}(y) & \text { on } B_{R+1}^{\rho_{m}}(p) \backslash B_{R}^{\rho_{m}}(p) \\ 0 & \text { on } M \backslash B_{R+1}^{\rho_{m}}(p) .\end{cases}
$$

By the weighted Poincaré inequality at infinity, we get

$$
\begin{aligned}
\left|\int_{\mathcal{L}_{p}(\delta \varepsilon, \varepsilon) \cap B_{R}^{\rho_{m}}(p)} G(p, y) f(y) \mathrm{d} y\right| & \leq \int_{\mathcal{L}_{p}(\delta \varepsilon, \varepsilon) \cap B_{R}^{\rho_{m}}(p)} G(p, y)|f(y)| \mathrm{d} y \\
& \leq \sup _{\mathcal{L}_{p}(\delta \varepsilon, \varepsilon) \cap B_{R}^{\rho_{m}}(p)} \frac{|f|}{\rho_{m}} \int_{M} \rho_{m}(y) G(p, y) \phi^{2}(y) \mathrm{d} y \\
& \leq \sup _{\mathcal{L}_{p}(\delta \varepsilon, \varepsilon) \cap B_{R}^{\rho_{m}}(p)} \frac{|f|}{\rho_{m}} \int_{M}|\nabla(\sqrt{G(p, y)} \phi(y))|^{2} \mathrm{~d} y .
\end{aligned}
$$

We estimate

$$
\begin{aligned}
\int_{M}|\nabla(\sqrt{G(p, y)} \phi(y))|^{2} \mathrm{~d} y & \leq \frac{1}{2} \int_{\mathcal{L}_{p}\left(\frac{\delta \varepsilon}{2}, 2 \varepsilon\right)} \frac{|\nabla G(p, y)|^{2}}{G(p, y)} \mathrm{d} y+2 \int_{M} G(p, y)|\nabla \phi|^{2} \mathrm{~d} y \\
& =C(-\log \delta+1)+2 \int_{M} G(p, y)|\nabla \phi|^{2} \mathrm{~d} y
\end{aligned}
$$

where we used Lemma 4.4 in the last equality. On the other hand,

$$
\begin{aligned}
\int_{M} G(p, y)|\nabla \phi|^{2} \mathrm{~d} y \leq & 2 \int_{M} G(p, y)|\nabla \chi|^{2} \psi^{2} \mathrm{~d} y+2 \int_{M} G(p, y)|\nabla \psi|^{2} \chi^{2} \mathrm{~d} y \\
\leq & 2(\log 2)^{2} \int_{\mathcal{L}_{p}\left(\frac{\delta \varepsilon}{2}, 2 \varepsilon\right)} \frac{|\nabla G(p, y)|^{2}}{G(p, y)} \mathrm{d} y \\
& +2 \int_{B_{R+1}^{\rho}(p) \backslash B_{R}^{\rho}(p)} \rho_{m}(y) G(p, y) \chi^{2} \mathrm{~d} y \\
\leq & C(-\log \delta+1)+\frac{4}{\delta \varepsilon} \int_{B_{R+1}^{\rho_{m}}(p) \backslash B_{R}^{\rho_{m}}(p)} \rho_{m}(y) G^{2}(p, y) \mathrm{d} y .
\end{aligned}
$$

Now we let $R \rightarrow \infty$ and use Lemma 4.5. The thesis now follows.

In the special case when $M$ is non-parabolic with positive minimal Green's function $G$ and with weight $\rho(x)=\frac{|\nabla G(p, x)|^{2}}{4 G^{2}(p, x)}$, we have the following refinement of Proposition 4.8. 
Proposition 4.9 Assume that $(M, g)$ is non-parabolic with positive minimal Green's function $G$ and with weight $\rho(x)=\frac{|\nabla G(p, x)|^{2}}{4 G^{2}(p, x)}$. Then, there exists a positive constant $C$ such that for any function $f$ and any $0<\delta<1, \varepsilon>0$ one has

$$
\left|\int_{\mathcal{L}_{p}(\delta \varepsilon, \varepsilon)} G(p, y) f(y) \mathrm{d} y\right| \leq C(-\log \delta) \sup _{\mathcal{L}_{p}(\delta \varepsilon, \varepsilon)} \frac{|f|}{\rho} .
$$

Proof We have

$$
\begin{aligned}
\left|\int_{\mathcal{L}_{p}(\delta \varepsilon, \varepsilon)} G(p, y) f(y) \mathrm{d} y\right| & \leq \sup _{\mathcal{L}_{p}(\delta \varepsilon, \varepsilon)} \frac{|f|}{\rho}\left(\int_{\mathcal{L}_{p}(\delta \varepsilon, \varepsilon)} G(p, y) \rho(y) \mathrm{d} y\right) \\
& =\frac{1}{4} \sup _{\mathcal{L}_{p}(\delta \varepsilon, \varepsilon)} \frac{|f|}{\rho}\left(\int_{\mathcal{L}_{p}(\delta \varepsilon, \varepsilon)} \frac{|\nabla G(p, y)|^{2}}{G(p, y)} \mathrm{d} y\right) \\
& =\frac{1}{4}(-\log \delta) \sup _{\mathcal{L}_{p}(\delta \varepsilon, \varepsilon)} \frac{|f|}{\rho},
\end{aligned}
$$

where we have used Lemma 4.4 in the last equality.

\section{Proof of Theorem 1.1}

In order to prove Theorem 1.1, we will show that

$$
|u(x)|=\left|\int_{M} G(x, y) f(y) \mathrm{d} y\right| \leq v(x),
$$

with $v \in C^{0}(M)$. We divide the proof in two parts, we first consider the case when $(M, g)$ is non-parabolic, and then, the case when it is parabolic.

Proof of Theorem 1.1 Case 1: $(M, g)$ non-parabolic.

By assumption, $(M, g)$ satisfies $\left(\mathcal{P}_{w}^{\infty}\right)$ w.r.t. the family of weights $\rho_{R}, R \geq R_{0}$. Let $x \in M$ and choose $R=R(x)>R_{0}$ large enough so that $x \in B_{R}(p)$. One has

$$
\begin{aligned}
\left|\int_{M} G(x, y) f(y) \mathrm{d} y\right| & \leq\left|\int_{B_{R}(p)} G(x, y) f(y) \mathrm{d} y\right|+\left|\int_{M \backslash B_{R}(p)} G(x, y) f(y) \mathrm{d} y\right| \\
& \leq C_{1}(x)+\int_{M \backslash B_{R}(p)} G(x, y)|f(y)| \mathrm{d} y
\end{aligned}
$$

since $G(x, \cdot) \in L_{\text {loc }}^{1}(M)$. Hence, by Harnack's inequality, we have

$$
\begin{aligned}
\left|\int_{M} G(x, y) f(y) \mathrm{d} y\right| & \leq C_{1}(x)+C_{2}(x) \int_{M \backslash B_{R}(p)} G(p, y)|f(y)| \mathrm{d} y \\
& \leq C_{1}(x)+C_{2}(x) \int_{M} G(p, y)|f(y)| \mathrm{d} y,
\end{aligned}
$$


where $C_{2}(x)$ can be chosen as the constant in the Harnack's inequality for the ball $B_{r(x)+1}(p)$. For any $a>0$, we estimate

$$
\begin{aligned}
\int_{M} G(p, y)|f(y)| \mathrm{d} y= & \int_{\mathcal{L}_{p}(0, A \exp (B \omega(a)))} G(p, y)|f(y)| \mathrm{d} y \\
& +\int_{\mathcal{L}_{p}(A \exp (B \omega(a)), \infty)} G(p, y)|f(y)| \mathrm{d} y .
\end{aligned}
$$

By Proposition 4.7, Remark 4.3 we get

$$
\int_{M} G(p, y)|f(y)| \mathrm{d} y \leq \int_{\mathcal{L}_{p}(0, A \exp (B \omega(a)))} G(p, y)|f(y)| \mathrm{d} y+C_{3}(a)
$$

for some positive constant $C_{3}(a)$. To estimate the first integral, we observe that for any $m_{0}=m_{0}(x) \geq a$ one has

$$
\begin{aligned}
& \int_{\mathcal{L}_{p}(0, A \exp (B \omega(a)))} G(x, y)|f(y)| \mathrm{d} y=\int_{\mathcal{L}_{p}\left(0,(2 A)^{-1} \exp \left(-B \omega\left(m_{0}\right)\right)\right)} G(x, y)|f(y)| \mathrm{d} y \\
& \quad+\int_{\mathcal{L}_{p}\left((2 A)^{-1} \exp \left(-B \omega\left(m_{0}\right)\right), A \exp (B \omega(a))\right)} G(x, y)|f(y)| \mathrm{d} y .
\end{aligned}
$$

We need the following lemma.

Lemma 5.1 Choose A, $B$ as in Lemma 4.1. For any $m \geq m_{0} \geq a$ one has

$$
\mathcal{L}_{p}\left(0, A^{-1} \exp (-B \omega(m))\right) \subset M \backslash B_{m}(p) .
$$

Proof Since $m_{0} \geq a$, Remark 4.2 implies

$$
\mathcal{L}_{p}\left(0, A^{-1} \exp \left(-B \omega\left(m_{0}\right)\right)\right) \subset \mathcal{L}_{p}\left(0, A^{-1} \exp (-B \omega(a))\right) \subset M \backslash B_{a}(p) .
$$

If

$$
z \in \mathcal{L}_{p}\left(0, A^{-1} \exp (-B \omega(m))\right) \subset M \backslash B_{a}(p),
$$

then by Lemma 4.1

$$
A^{-1} \exp (-B \omega(m)) \geq G(p, z) \geq A^{-1} \exp (-B \omega(z)) .
$$

Thus,

$$
\omega(z) \geq \omega(m)
$$

and, by monotonicity of $\omega$, we obtain $r(z) \geq m$.

In particular, we get

$$
\mathcal{L}_{p}\left(0,(2 A)^{-1} \exp \left(-B \omega\left(m_{0}\right)\right)\right) \subset \mathcal{L}_{p}\left(0, A^{-1} \exp \left(-B \omega\left(m_{0}\right)\right)\right) \subset M \backslash B_{m_{0}}(p) .
$$

Thus, 


$$
\mathcal{L}_{p}\left((2 A)^{-1} \exp \left(-B \omega\left(m_{0}\right)\right), A \exp (B \omega(a))\right) \subset B_{m_{0}}(p)
$$

Then, since $G(x, \cdot) \in L_{\text {loc }}^{1}(M)$, we get

$$
\int_{\mathcal{L}_{p}\left((2 A)^{-1} \exp \left(-B \omega\left(m_{0}\right)\right), A \exp (B \omega(a))\right)} G(x, y)|f(y)| \mathrm{d} y \leq C_{4}\left(a, m_{0}\right) .
$$

Now, for any $m \geq m_{0}$, let

$$
\varepsilon:=(2 A)^{-1} \exp (-B \omega(m)), \quad \delta:=\exp (B \omega(m)-B \omega(m+1)) .
$$

By Lemma 5.1,

$$
\mathcal{L}_{p}(0,2 \varepsilon) \subset M \backslash B_{m}(p) .
$$

Hence, we can apply Proposition 4.8 obtaining

$$
\begin{aligned}
& \int_{\mathcal{L}_{p}\left(0,(2 A)^{-1} \exp \left(-B \omega\left(m_{0}\right)\right)\right)} G(x, y)|f(y)| \mathrm{d} y \\
& \quad=\sum_{m \geq m_{0}} \int_{\mathcal{L}_{p}\left((2 A)^{-1} \exp (-B \omega(m+1)),(2 A)^{-1} \exp (-B \omega(m))\right)} G(x, y)|f(y)| \mathrm{d} y \\
& \quad \leq C \sum_{m \geq m_{0}}^{\infty}(\omega(m+1)-\omega(m)+1) \sup _{\mathcal{L}_{p}\left((2 A)^{-1} \exp (-B \omega(m+1)),(2 A)^{-1} \exp (-B \omega(m))\right)} \frac{|f|}{\rho_{m}} \\
& \quad \leq C \sum_{m \geq m_{0}}^{\infty}(\omega(m+1)-\omega(m)+1) \sup _{\mathcal{L}_{p}\left(0, A^{-1} \exp (-B \omega(m))\right)} \frac{|f|}{\rho_{m}} \\
& \quad \leq C \sum_{m \geq m_{0}}^{\infty}(\omega(m+1)-\omega(m)+1) \sup _{M \backslash B_{m}(p)} \frac{|f|}{\rho_{m}}<\infty,
\end{aligned}
$$

where in the last inequality we used Lemma 5.1. The proof of Theorem 1.1 is complete in this case.

Case 2: $(M, g)$ parabolic.

Let $G(x, y)$ be a Green's function on $M$ (which is positive inside a certain ball, and negative outside). Fix any $R>0$ and let $\rho \equiv \rho_{R_{0}}$. Note that arguing as in the proof of (8), it is sufficient to estimate

$$
\begin{aligned}
\int_{M}|G(p, y)||f(y)| \mathrm{d} y & =\int_{M \backslash B_{R}^{\rho}(p)}|G(p, y)||f(y)| \mathrm{d} y+\int_{B_{R}^{\rho}(p)}|G(p, y)||f(y)| \mathrm{d} y \\
& \leq \int_{M \backslash B_{R}^{\rho}(p)}|G(p, y)||f(y)| \mathrm{d} y+C,
\end{aligned}
$$

since $G(p, \cdot) \in L_{\text {loc }}^{1}(M)$ and $f$ is locally bounded. We have that

$$
M \backslash B_{R}^{\rho}(p)=\bigcup_{i=1}^{N} E_{i},
$$

where each $E_{i}$ is an end with respect to $B_{R}^{\rho}(p)$. Note that every end $E_{i}$ is parabolic. In fact, if at least one end $E_{i}$ is non-parabolic, then $(M, g)$ is non-parabolic (see [8] for a nice 
overview), but we are in the case that $(M, g)$ is parabolic. Since every $E_{i}$ is parabolic, every $E_{i}$ has finite weighted volume (see [9]), i.e.,

$$
\int_{E_{i}} \rho \mathrm{d} y<\infty .
$$

Now choose $R$ large enough so that we can apply Lemma 4.5 obtaining

$$
\begin{aligned}
& \int_{M \backslash B_{R}^{\rho}(p)}|G(p, y)||f(y)| \mathrm{d} y \\
& \quad \leq\left(\int_{M \backslash B_{R}^{\rho}(p)} \rho(y)|G(p, y)|^{2} \mathrm{~d} y\right)^{\frac{1}{2}}\left(\int_{M \backslash B_{R}^{\rho}(p)} \rho(y)\left(\frac{|f(y)|}{\rho(y)}\right)^{2} \mathrm{~d} y\right)^{\frac{1}{2}} \\
& \quad \leq C \sup _{M \backslash B_{R_{0}}(p)} \frac{|f|}{\rho} \int_{M \backslash B_{R}^{\rho}(p)} \rho \mathrm{d} y<\infty .
\end{aligned}
$$

This concludes the proof of Theorem 1.1.

Proof of Theorem 1.2 We start as in the proof of Theorem 1.1 using (8), (9), (10) and (13). Then, similar to (15), using Proposition 4.9, we obtain

$$
\begin{aligned}
& \int_{\mathcal{L}_{p}\left(0,(2 A)^{-1} \exp \left(-B \omega\left(m_{0}\right)\right)\right)} G(x, y)|f(y)| \mathrm{d} y \\
& \quad=\sum_{m \geq m_{0}} \int_{\mathcal{L}_{p}\left((2 A)^{-1} \exp (-B \omega(m+1)),(2 A)^{-1} \exp (-B \omega(m))\right)} G(x, y)|f(y)| \mathrm{d} y \\
& \quad \leq C \sum_{m \geq m_{0}}^{\infty}(\omega(m+1)-\omega(m)) \sup _{M \backslash B_{m}(p)} \frac{|f|}{\rho}<\infty,
\end{aligned}
$$

Then

$$
\left|\int_{M} G(x, y) f(y) \mathrm{d} y\right|<\infty
$$

and the proof of Theorem 1.2 is complete.

\section{Cartan-Hadamard and model manifolds}

We consider Cartan-Hadamard manifolds, i.e., complete, non-compact, simply connected Riemannian manifolds with non-positive sectional curvatures everywhere. Observe that on Cartan-Hadamard manifolds the cut locus of any point $p$ is empty. Hence, for any $x \in M \backslash\{p\}$ one can define its polar coordinates with pole at $p$, namely $r(x)=\operatorname{dist}(x, p)$ and $\theta \in \mathbb{S}^{n-1}$. We have 


$$
\operatorname{meas}\left(\partial B_{r}(p)\right)=\int_{\mathbb{S}^{n-1}} A(r, \theta) \mathrm{d} \theta^{1} d \theta^{2} \ldots \mathrm{d} \theta^{n-1}
$$

for a specific positive function $A$ which is related to the metric tensor [6, Sect. 3]. Moreover, it is direct to see that the Laplace-Beltrami operator in polar coordinates has the form

$$
\Delta=\frac{\partial^{2}}{\partial r^{2}}+m(r, \theta) \frac{\partial}{\partial r}+\Delta_{\theta},
$$

where $m(r, \theta):=\frac{\partial}{\partial r}(\log A)$ and $\Delta_{\theta}$ is the Laplace-Beltrami operator on $\partial B_{r}(p)$. We have

$$
m(r, \theta)=\Delta r(x) .
$$

Let

$$
\mathcal{A}:=\left\{f \in C^{\infty}((0, \infty)) \cap C^{1}([0, \infty)): f^{\prime}(0)=1, f(0)=0, f>0 \text { in }(0, \infty)\right\} .
$$

We say that $(M, g)$ is a rotationally symmetric manifold or a model manifold if the Riemannian metric is given by

$$
g=\mathrm{d} r^{2}+\varphi(r)^{2} \mathrm{~d} \theta^{2},
$$

where $\mathrm{d} \theta^{2}$ is the standard metric on $\mathbb{S}^{n-1}$ and $\varphi \in \mathcal{A}$. In this case,

$$
\Delta=\frac{\partial^{2}}{\partial r^{2}}+(n-1) \frac{\varphi^{\prime}}{\varphi} \frac{\partial}{\partial r}+\frac{1}{\varphi^{2}} \Delta_{\mathbb{S}^{n-1}} .
$$

Note that $\varphi(r)=r$ corresponds to $M=\mathbb{R}^{n}$, while $\varphi(r)=\sinh r$ corresponds to $M=\mathbb{H}^{n}$, namely the $n$-dimensional hyperbolic space. The Ricci curvature in the radial direction is given by

$$
\operatorname{Ric}(\nabla r, \nabla r)(x)=-(n-1) \frac{\varphi^{\prime \prime}(r(x))}{\varphi(r(x))} .
$$

\subsection{Cartan-Hadamard manifolds}

Concerning the validity of the property $\left(\mathcal{P}_{w}^{\infty}\right)$ w.r.t. the family of weights $\rho_{R}, R \geq R_{0}$ on a Cartan-Hadamard manifold we have the following result.

Lemma 6.1 Let $(M, g)$ be a Cartan-Hadamard manifold with

$$
\operatorname{Ric}(\nabla r, \nabla r)(x) \leq-C(1+r(x))^{\gamma}
$$

for some $\gamma \in \mathbb{R}, C>0$ and any $x \in M \backslash\{p\}$. Then $(M, g)$ satisfies the property $\left(\mathcal{P}_{w}^{\infty}\right)$ with

$$
\rho_{R}(x)= \begin{cases}C^{\prime} r(x)^{\gamma} & \text { if } \gamma \geq-2 \\ C^{\prime} r(x)^{-2} & \text { if } \gamma<-2\end{cases}
$$

for all $R>0$ large enough and some $C^{\prime}>0$. 
Remark 6.2 As it will be clear from the proof, we have a weighted Poincaré inequality on $M$ if $\gamma \leq 0$ and a the weighted Poincare inequality for functions with compact support in $M \backslash B_{1}(p)$ if $\gamma>0$.

Proof We can find $\varphi \in \mathcal{A}$ given by

$$
\varphi(r)= \begin{cases}\exp \left(B r^{1+\frac{\gamma}{2}}\right) & \text { if } \gamma>-2 \\ r^{\delta} & \text { if } \gamma=-2 \\ r & \text { if } \gamma<-2\end{cases}
$$

for $r$ large enough, $B>0$ small, $\delta=\delta(C)>1$ such that $\operatorname{Ric}(\nabla r, \nabla r)(x) \leq-\frac{\varphi^{\prime \prime}(r(x))}{\varphi(r(x))}$. By the Laplacian comparison in a strong form, which is valid only on Cartan-Hadamard manifolds (see [18, Theorem 2.15]), one has

$$
\Delta r(x) \geq \begin{cases}C r(x)^{\gamma / 2} & \text { if } \gamma \geq-2 \\ C r(x)^{-1} & \text { if } \gamma<-2\end{cases}
$$

Suppose $\gamma \leq 0$ and let $\alpha:=\max \{\gamma,-2\} \leq 0$. For any $u \in C_{c}^{\infty}(M)$, since $|\nabla r|^{2}=1$, we have

$$
\begin{aligned}
& C \int_{M} r(y)^{\alpha} u(y)^{2} \mathrm{~d} y \\
& \quad \leq \int_{M} u(y)^{2} r(y)^{\alpha / 2} \Delta r(y) \mathrm{d} y \\
& \quad=-2 \int_{M}\langle\nabla u, \nabla r\rangle u(y) r(y)^{\alpha / 2} \mathrm{~d} y+\frac{\alpha}{2} \int_{M} u(y)^{2} r(y)^{\alpha / 2-1}|\nabla r(y)|^{2} \mathrm{~d} y \\
& \quad \leq 2 \int_{M}|u(y)||\nabla u(y)| r(y)^{\alpha / 2} \mathrm{~d} y \\
& \quad \leq \frac{C}{2} \int_{M} r(y)^{\alpha} u(y)^{2} \mathrm{~d} y+\frac{2}{C} \int_{M}|\nabla u(y)|^{2} \mathrm{~d} y .
\end{aligned}
$$

Thus,

$$
\int_{M} r(y)^{\alpha} u(y)^{2} \mathrm{~d} y \leq \frac{4}{C^{2}} \int_{M}|\nabla u(y)|^{2} \mathrm{~d} y
$$

and the weighted Poincare inequality on $M$ follows in this case.

Suppose now $\gamma>0$. By a Barta-type argument (see, e.g., [7, Theorem 11.17]),

$$
\lambda_{1}\left(M \backslash B_{R}(p)\right) \geq\left[C R^{\frac{\gamma}{2}}\right]^{2} \quad \text { in } M \backslash B_{R}(p) .
$$

Thus, the Poincaré inequality reads

$$
C R^{\gamma} \int_{M} u(y)^{2} \mathrm{~d} y \leq \int_{M}|\nabla u(y)|^{2} \mathrm{~d} y
$$

for any $u$ with compact support in $M \backslash B_{R}(p)$. Now let $R>1$ and, for every $k \in \mathbb{N}$, define the cutoff functions 


$$
\varphi_{k}(x):= \begin{cases}r(x)-k+1, & r(x) \in[k-1, k) \\ k+1-r(x), & r(x) \in[k, k+1) \\ 0 & \text { otherwise. }\end{cases}
$$

Note that $\left|\nabla \varphi_{k}\right| \leq 1$ and for all $x \in M \backslash B_{1}(p), \sum_{k} \varphi_{k}=1$ and $x \in \operatorname{supp} \varphi_{k}$ at most for two integers $k$. If supp $u \subset M \backslash B_{1}(p)$, we have

$$
\begin{aligned}
\int_{M} r(y)^{\gamma} u(y)^{2} \mathrm{~d} y & =\int_{M} r(y)^{\gamma}\left(\sum_{k} \varphi_{k}(y) u(y)\right)^{2} \mathrm{~d} y \\
& \leq 2 \sum_{k} \int_{M} r(y)^{\gamma} \varphi_{k}(y)^{2} u(y)^{2} \mathrm{~d} y \\
& \leq C \sum_{k}(k-1)^{\gamma} \int_{M} \varphi_{k}(y)^{2} u(y)^{2} \mathrm{~d} y \\
& \leq C \sum_{k} \int_{M}\left|\nabla\left(\varphi_{k}(y) u(y)\right)\right|^{2} \mathrm{~d} y,
\end{aligned}
$$

where in the last passage we used (17) with $R=k-1$. Thus,

$$
\begin{aligned}
\int_{M} r(y)^{\gamma} u(y)^{2} \mathrm{~d} y & \leq C \sum_{k}\left(\int_{M} u(y)^{2}\left|\nabla \varphi_{k}(y)\right|^{2} \mathrm{~d} y+\int_{M} \varphi_{k}(y)^{2}|\nabla u(y)|^{2} \mathrm{~d} y\right) \\
& \leq C \int_{M} u(y)^{2} \mathrm{~d} y+C \int_{M}|\nabla u(y)|^{2} \mathrm{~d} y \\
& \leq C \int_{M}|\nabla u(y)|^{2} \mathrm{~d} y,
\end{aligned}
$$

where in the last passage we used (17) with $R=1$. Hence, the weighted Poincare inequality holds for functions with support in $M \backslash B_{1}(p)$.

Finally, the completeness of the metric $g_{\rho_{R}}:=\rho_{R} g$ follows. In fact, for any curve $\eta(s)$ parametrized by arclength with $0 \leq s \leq T$, the length of $\eta$ with respect tp $g_{\rho_{R}}$ is given by

$$
\int_{\eta} \sqrt{\rho_{R}} \mathrm{~d} s \rightarrow \infty \quad \text { as } T \rightarrow \infty .
$$

Let us write some estimates which will be useful both in the proof of Corollary 1.4 and in Sect. 6.2. Choose $\varphi \in \mathcal{A}$ as in (16) with $\gamma=\gamma_{1}$ obtaining

$$
\frac{\varphi^{\prime}(r(x))}{\varphi(r(x))}= \begin{cases}C r(x)^{\gamma_{1} / 2} & \text { if } \gamma_{1} \geq-2 \\ C r(x)^{-1} & \text { if } \gamma_{1}<-2\end{cases}
$$

and

$$
\frac{\varphi^{\prime \prime}(r(x))}{\varphi(r(x))}= \begin{cases}C r(x)^{\gamma_{1}}+C^{\prime} r(x)^{\gamma_{1} / 2-1} & \text { if } \gamma_{1} \geq-2 \\ 0 & \text { if } \gamma_{1}<-2\end{cases}
$$

for $r(x)>R>1$. A simple computation shows that for $R=r(x) / 4$, one has 


$$
\begin{aligned}
& K_{R}(x)= \begin{cases}C r(x)^{\gamma_{1} / 2} & \text { if } \gamma_{1} \geq-2 \\
0 & \text { if } \gamma_{1}<-2,\end{cases} \\
& \frac{I_{R}(x)}{R}= \begin{cases}C r(x)^{\gamma_{1} / 2-1} \operatorname{coth}\left(C^{\prime} r(x)^{\gamma_{1} / 2+1}\right) & \text { if } \gamma_{1} \geq-2 \\
\frac{2}{r(x)^{2}} & \text { if } \gamma_{1}<-2\end{cases}
\end{aligned}
$$

and

$$
Q_{R}(x)= \begin{cases}C r(x)^{\gamma_{1}} & \text { if } \gamma_{1} \geq-2 \\ \frac{2}{r(x)^{2}} & \text { if } \gamma_{1}<-2\end{cases}
$$

Thus,

$$
\omega(r)= \begin{cases}C r^{\gamma_{1} / 2+1} & \text { if } \gamma_{1} \geq-2 \\ C \log r & \text { if } \gamma_{1}<-2\end{cases}
$$

and, as $m \rightarrow \infty$,

$$
\omega(m+1)-\omega(m) \sim \begin{cases}C m^{\gamma_{1} / 2} & \text { if } \gamma_{1} \geq-2 \\ C m^{-1} & \text { if } \gamma_{1}<-2\end{cases}
$$

On the other hand, using Lemma 6.1 with $\gamma=\gamma_{2}$, we get the estimate

$$
\sup _{M \backslash B_{m}(p)} \frac{1}{\rho_{m}} \leq\left\{\begin{array}{ll}
C m^{-\gamma_{2}} & \text { if } \gamma_{2} \geq-2 \\
C m^{2} & \text { if } \gamma_{2}<-2
\end{array} .\right.
$$

Proof of Corollary 1.4 For $\gamma_{1} \geq \gamma_{2}$ and $\gamma_{1} \geq 0$, we get

$$
\sum_{m}^{\infty}(\omega(m+1)-\omega(m)+1) \sup _{M \backslash B_{m}(p)} \frac{|f|}{\rho_{m}} \leq \begin{cases}C \sum_{m}^{\infty} m^{\gamma_{1} / 2-\gamma_{2}-\alpha} & \text { if } \gamma_{2} \geq-2 \\ C \sum_{m}^{\infty} m^{2+\gamma_{1} / 2-\alpha} & \text { if } \gamma_{2}<-2 .\end{cases}
$$

and the thesis immediately follows.

\subsection{Optimality on rotationally symmetric manifolds}

We show that the assumptions in Theorem 1.2 are sharp on model manifolds. Let $(M, g)$ be a rotationally symmetric manifold with $\varphi \in \mathcal{A}$ defined as in (16) for any $r>1$. One has

$$
\int_{M} G(x, y) f(y) \mathrm{d} y<\infty \quad \text { for any } x \in M \quad \Longleftrightarrow \quad \int_{M} G(p, y) f(y) \mathrm{d} y<\infty .
$$

Hence, a solution of $-\Delta u=f$ in $M$ exists if and only if

$$
u(p)=\int_{0}^{\infty}\left(\int_{r}^{\infty} \frac{1}{\varphi(t)^{n-1}} \mathrm{~d} t\right) f(r) \varphi(r)^{n-1} \mathrm{~d} r<\infty .
$$

Case 1: $\gamma>-2$. With our choice of $\varphi$, by the change of variable $s=t^{1+\frac{\gamma}{2}}$, it is easily seen that for any $r>0$ sufficiently large 


$$
\int_{r}^{\infty} \frac{1}{\varphi(t)^{n-1}} \mathrm{~d} t \sim C r^{-\frac{\gamma}{2}} \exp \left(-(n-1) r^{1+\frac{\gamma}{2}}\right)
$$

Hence,

$$
\begin{aligned}
& \frac{1}{C} \int_{1}^{\infty} r^{-\frac{\gamma}{2}} \exp \left(-(n-1) r^{1+\frac{\gamma}{2}}\right) \frac{1}{(1+r)^{\alpha}} \exp \left((n-1) r^{1+\frac{\gamma}{2}}\right) \mathrm{d} r \leq|u(p)| \\
& \quad \leq C \int_{1}^{\infty} r^{-\frac{\gamma}{2}} \exp \left(-(n-1) r^{1+\frac{\gamma}{2}}\right) \frac{1}{(1+r)^{\alpha}} \exp \left((n-1) r^{1+\frac{\gamma}{2}}\right) \mathrm{d} r
\end{aligned}
$$

Therefore,

$$
\frac{1}{C} \int_{1}^{\infty} \frac{1}{r^{\alpha+\frac{\gamma}{2}}} \mathrm{~d} r \leq|u(p)| \leq C \int_{1}^{\infty} \frac{1}{r^{\alpha+\frac{\gamma}{2}}} \mathrm{~d} r .
$$

This yields that

$$
|u(p)|<\infty \quad \text { if and only if } \quad \alpha>1-\frac{\gamma}{2} .
$$

On the other hand, a direct computation, using (19), shows that

$$
\rho(x)=\frac{|\nabla G(p, x)|^{2}}{4 G^{2}(p, x)} \sim C r(x)^{\gamma} .
$$

Furthermore, from (18), the assumption of Theorem 1.2 is satisfied if and only if

$$
\alpha>1-\frac{\gamma}{2},
$$

and the optimality follows in this case.

Case 2: $\gamma=-2$. We have,

$$
\int_{r}^{\infty} \frac{1}{\varphi(t)^{n-1}} \mathrm{~d} t=C r^{-\delta(n-1)+1} .
$$

Thus,

$$
\frac{1}{C} \int_{1}^{\infty} r^{-\delta(n-1)+1} \frac{1}{(1+r)^{\alpha}} r^{\delta(n-1)} \mathrm{d} r \leq|u(p)| \leq C \int_{1}^{\infty} r^{-\delta(n-1)+1} \frac{1}{(1+r)^{\alpha}} r^{\delta(n-1)} \mathrm{d} r
$$

Therefore,

$$
\frac{1}{C} \int_{1}^{\infty} \frac{1}{r^{\alpha-1}} \mathrm{~d} r \leq|u(p)| \leq C \int_{1}^{\infty} \frac{1}{r^{\alpha-1}} \mathrm{~d} r
$$

and

$$
|u(p)|<\infty \quad \text { if and only if } \quad \alpha>2 .
$$

On the other hand, a direct computation, using (20), shows that 


$$
\rho(x)=\frac{|\nabla G(p, x)|^{2}}{4 G^{2}(p, x)} \sim C r(x)^{-2} .
$$

Furthermore, from (18), the assumption of Theorem 1.2 is satisfied if and only if

$$
\alpha>2,
$$

and the optimality follows in this case.

Case 3: $\gamma<-2$. We have,

$$
\int_{r}^{\infty} \frac{1}{\varphi(t)^{n-1}} \mathrm{~d} t=C r^{2-n}
$$

Thus,

$$
\frac{1}{C} \int_{1}^{\infty} r^{2-n} \frac{1}{(1+r)^{\alpha}} r^{n-1} \mathrm{~d} r \leq|u(p)| \leq C \int_{1}^{\infty} r^{2-n} \frac{1}{(1+r)^{\alpha}} r^{n-1} \mathrm{~d} r
$$

Therefore,

$$
\frac{1}{C} \int_{1}^{\infty} \frac{1}{r^{\alpha-1}} \mathrm{~d} r \leq|u(p)| \leq C \int_{1}^{\infty} \frac{1}{r^{\alpha-1}} \mathrm{~d} r
$$

and

$$
|u(p)|<\infty \quad \text { if and only if } \quad \alpha>2 .
$$

On the other hand, a direct computation, using (21), shows that

$$
\rho(x)=\frac{|\nabla G(p, x)|^{2}}{4 G^{2}(p, x)} \sim C r(x)^{-2} .
$$

Furthermore, from (18), the assumption of Theorem 1.2 is satisfied if and only if

$$
\alpha>2 \text {, }
$$

and the optimality follows in this last case.

Acknowledgements Open access funding provided by Politecnico di Milano within the CRUI-CARE Agreement. The authors are members of the Gruppo Nazionale per l'Analisi Matematica, la Probabilità e le loro Applicazioni (GNAMPA) of the Istituto Nazionale di Alta Matematica (INdAM). The first two authors are supported by the PRIN-2015KB9WPT project "Variational methods, with applications to problems in mathematical physics and geometry." The third author is supported by the PRIN-201758MTR2 project "Direct and inverse problems for partial differential equations: theoretical aspects and applications."

Open Access This article is licensed under a Creative Commons Attribution 4.0 International License, which permits use, sharing, adaptation, distribution and reproduction in any medium or format, as long as you give appropriate credit to the original author(s) and the source, provide a link to the Creative Commons licence, and indicate if changes were made. The images or other third party material in this article are included in the article's Creative Commons licence, unless indicated otherwise in a credit line to the material. If material is not included in the article's Creative Commons licence and your intended use is not permitted by statutory regulation or exceeds the permitted use, you will need to obtain permission directly from the copyright holder. To view a copy of this licence, visit http://creativecommons.org/licenses/by/4.0/. 


\section{References}

1. Berchio, E., Ganguly, D., Grillo, G.: Sharp Poincaré-Hardy and Poincaré-Rellich inequalities on the hyperbolic space. J. Funct. Anal. 272, 1661-1703 (2017)

2. Calabi, E.: An extension of E. Hopf's maximum principle with application to Riemannian geometry. Duke Math. J. 25, 45-46 (1958)

3. Carron, G.: Inegalites de Hardy sur les varietes Riemanniennes non-compactes. J. Math. Pures Appl. 76, 883-891 (1997)

4. Catino, G., Monticelli, D.D., Punzo, F.: The Poisson equation on manifolds with positive essential spectrum. Calc. Var. Part. Differ. Equ. 58(4), 146 (2019)

5. Cheng, S.Y., Yau, S.T.: Differential equations on Riemannian manifolds and their geometric applications. Commun. Pure Appl. Math. 28, 333-354 (1975)

6. Grigor'yan, A.: Analytic and geometric background of recurrence and non-explosion of the Brownian motion on Riemannian manifolds. Bull. Am. Math. Soc. 36, 135-249 (1999)

7. Grigor'yan, A.: Heat Kernel and Analysis on Manifolds, AMS/IP Studies in Advanced Mathematics, vol. 47, American Mathematical Society, Providence, RI. International Press, Boston, MA (2009)

8. Li, P.: Curvature and function theory on Riemannian manifolds. Surv. Differ. Geom., 7, Int. Press, Somerville, MA, 375-432 (2000)

9. Li, P., Wang, J.: Complete manifolds with positive spectrum. J. Differ. Geom. 58(3), 501-534 (2001)

10. Li, P., Wang, J.: Weighted Poincaré inequality and rigidity of complete manifolds. Ann. Sc. Ec. Norm. Sup., 4e serie, t. 39, 921-982 (2006)

11. Malgrange, B.: Existence et approximation des solutions de equations aux derivees partielles et des equations de convolution. Annales de l'Inst. Fourier 6, 271-355 (1955)

12. Munteanu, O., Sesum, N.: The Poisson equation on complete manifolds with positive spectrum and applications. Adv. Math. 223(1), 198-219 (2010)

13. Munteanu, O., Sung, C.-J.A., Wang, J.: Poisson equation on complete manifolds. Adv. Math. 348, 81-145 (2019)

14. Munteanu, O., Sung, C.-J.A., Wang, J.: Weighted Poincaré inequality and the Poisson equation. Preprint 2019. arXiv:1904.13337

15. Ni, L.: The Poisson equation and Hermitian Einstein metrics on Holomorphic vector bundles over complete noncompact Kähler manifolds. Indiana Univ. Math. J. 51, 670-703 (2002)

16. Ni, L., Shi, Y., Tam, L.F.: Poisson equation, Poincaré-Lelong equation and curvature decay on complete Kähler manifolds. J. Differ. Geom. 57, 339-388 (2001)

17. Strichartz, R.: Analysis of the Laplacian on the complete Riemannian manifold. J. Funct. Anal. 52, 48-79 (1983)

18. Xin, Y.L.: Geometry of Harmonic Maps. Birkhäuser, Boston (1996)

19. Wang, J.: The spectrum of the Laplacian on a manifold of nonnegative Ricci curvature. Math. Res. Lett. 4, 473-479 (1997)

Publisher's Note Springer Nature remains neutral with regard to jurisdictional claims in published maps and institutional affiliations. 